\title{
Les sédiments dans les torrents de l'Arve : discontinuité fonctionnelle et impacts de l'aménagement des bassins versants
}

\author{
J.-L. Peiry \\ Département de Géographie, Université Lyon 3 \\ 74, rue Pasteur, 69239 Lyon Cedex
}

\section{Introduction}

La dynamique des sédiments de la plupart des rivières intramontagnardes alpestres est profondément perturbée par les interventions anthropiques (BLANCHET et BRISSaut, 1968 ; Blanic et Verdet, 1975 ; Peiry, 1987 ; Peiry et Bravard, ce volume...). Ces perturbations, telles les extractions de gravier dans le lit, les retenues hydroélectriques qui modifient le régime et bloquent une partie de la charge, ont pour conséquence une tendance quasigénéralisée à l'abaissement des lits fluviaux. La recherche des causes de l'incision des rivières alpestres nécessite que l'on s'interroge sur l'éventuelle responsabilité d'une diminution de la charge sédimentaire alimentant les axes de drainage de vallée, et plus particulièrement de celle fournie par les torrents.

Cette étude a pour double objectif de mieux appréhender la dynamique des sédiments torrentiels et de déterminer quels ont été les effets des travaux de correction entrepris depuis la fin du XIX ${ }^{\mathrm{e}}$ siècle sur cette dynamique. Elle résulte de l'analyse du comportement de huit bassins torrentiels de l'Arve supérieure et de ses affluents choisis pour leur appartenance à une large gamme d'environnements (fig. I. tabl. l). Ces torrents ont pour point commun de drainer des bassins en forte pente, situés dans des formations géologiques susceptibles de délivrer des débris en abondance. De plus, ils ont pour particularité d'être ou d'avoir été parmi les plus actifs de la vallée et d'avoir été aménagés à des degrés divers depuis 1890. Ils sont bien connus du Service de Restauration des Terrains en Montagne de la Haute-Savoie qui dispose pour chacun d'entre eux d'une abondante documentation que l'on a analysée.

\section{La dynamique des sédiments torrentiels}

La dynamique des sédiments torrentiels est caractérisée par la discontinuité entre les phénomènes majeurs d'alimentation en matériaux du thalweg et les remaniements sédimentaires d'origine torrentielle modifiant de manière significative la géomorphologie du chenal : de longues périodes de faible activité torrentielle, au cours desquelles les matériaux s'accumulent dans les lits, sont entrecoupées de phases de remaniement sédimentaire, brèves mais extrêmement intenses, mettant en jeu des forces considérables.

\subsection{La période d'accumulation sédimentaire}

Comme l'a souligné TrICART (1960), les versants évoluent la plupart du temps " par le haut ", leur dynamique n'étant pas forcément conditionnée par l'évolution des thalwegs : nombreux sont les matériaux mobilisés par des processus de versant, puis abandonnés sur les pentes, à l'écart de toute zone d'activité torrentielle. Les processus de versant entraînant les matériaux vers les thalwegs ont une origine variée; ils fonctionnent chacun à un rythme qui leur est propre, sur des pas de temps différents, souvent indépendamment des processus torrentiels, et la plupart du temps se relaient pour acheminer les matériaux jusqu'aux thalwegs. L'étude des torrents de la Haute-Arve à travers l'analyse des archives du service RTM de Haute-Savoie, complétée d'observations de terrain, a permis d'établir quels sont les principaux types de processus en activité :

1) Les érosions superficielles: acheminement d'importantes quantités de débris jusqu'aux thalwegs : le ravine-

\section{Sediments in the Arve torrents : functional discontinuities and impacts of improvement works on watersheds}

The sediment dynamics on torrents are marked by functional discontinuities : firstly, a long period of sedimentary accumulation in the channel; secondly, a brief period of sedimentary expulsion during density flows, which carry out large volumes of debris and change strongly the channel geomorphology. Since the end of the 19th century, man has attempted to control the frequency and the intensity of sedimentary transportation by improvement works on slopes and in the channel; they have favoured the retention of the load in the mountain and have diminished the frequency of debris flows. 


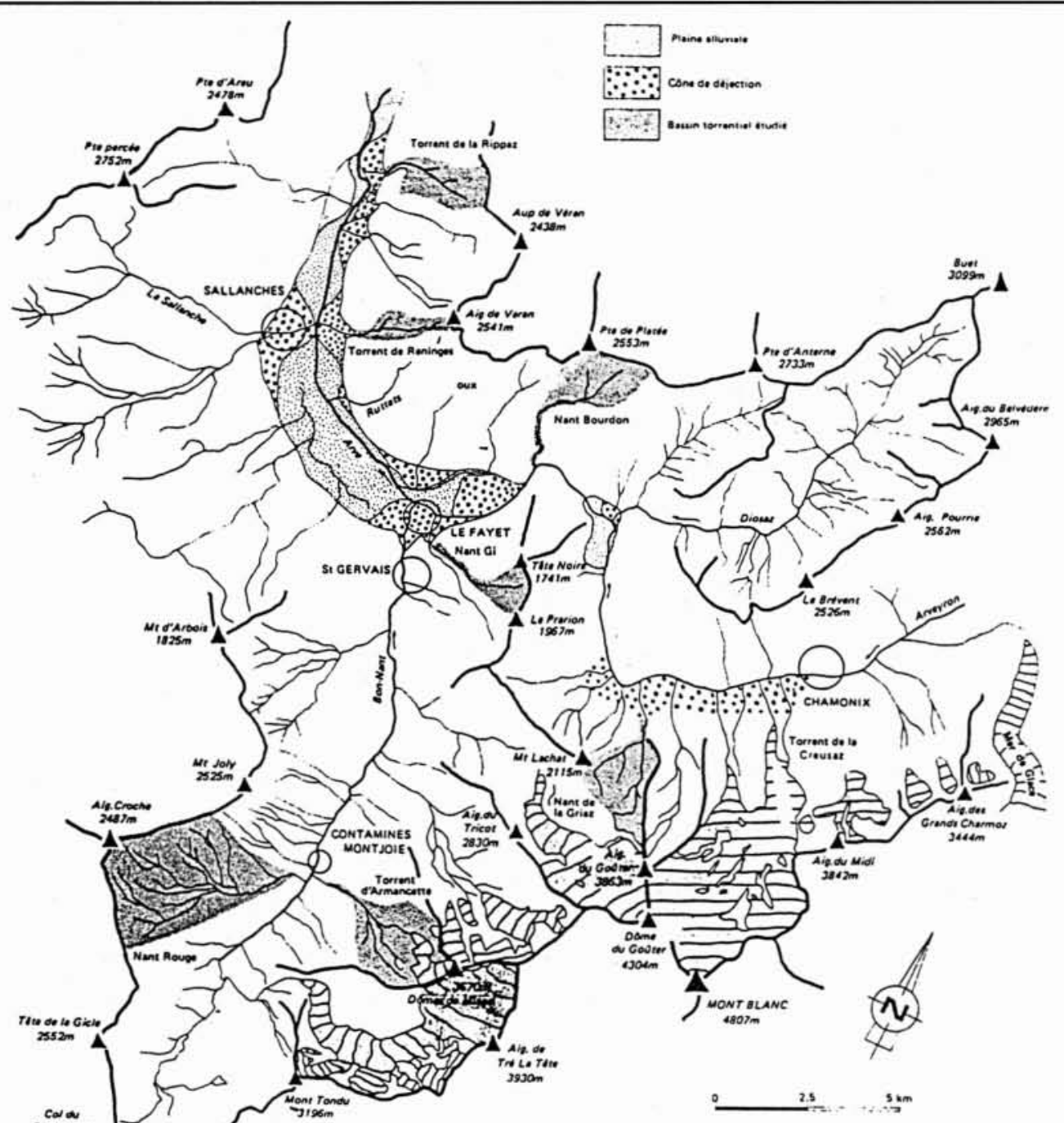

1. Localisation des bassins torrentiels étudiés.

Iableau 1: Car actér istiques des torrents étudiés.

\begin{tabular}{|c|c|c|c|c|c|}
\hline Torrents & $\begin{array}{l}\text { Surfoce } \\
\text { du bassin }\end{array}$ & Orientotion & $\begin{array}{r}\text { Altitude } \\
\text { moyenne }\end{array}$ & $\begin{array}{l}\text { Toux } \\
\text { Genglocement }\end{array}$ & Localisation \\
\hline Glociaire & . & & & & \\
\hline $\begin{array}{c}\text { Creusaz } \\
\text { (glocier des Bossons) }\end{array}$ & ) & N & $2500 \mathrm{~m}$ &, $50 \pi$ & Chamonix \\
\hline \multicolumn{6}{|l|}{ Mixte } \\
\hline Armancette & 745 he & 0 & $2300 \mathrm{~m}$ & $13 \%$ & $\begin{array}{l}\text { Contamines } \\
\text { - Montjole }\end{array}$ \\
\hline Griez & 420 no & $\mathrm{N}$ & $2200 \mathrm{~m}$ & 128 & Les Houches \\
\hline \multicolumn{6}{|l|}{$\begin{array}{l}\text { Sons influence } \\
\text { glacialre }\end{array}$} \\
\hline Nant Rouge & 1140 ho & E & $1750 \mathrm{~m}$ & 08 & $\begin{array}{l}\text { Contamines } \\
\text {-Montjole }\end{array}$ \\
\hline Nant Bourdon & 340 no & s & $1600 \mathrm{~m}$ & $0 \%$ & Plateau đ'Assy \\
\hline Reninges & 165 ha & so & $1500 \mathrm{~m}$ & $0 \%$ & Sallanches \\
\hline Nent Gibloux & 160 ho & 0 & $1.450 \mathrm{~m}$ & $0 \%$ & St-Gervais \\
\hline Rippoz & 255 ho & 0 & $1350 \mathrm{~m}$ & $0 \%$ & $\begin{array}{l}\text { Cluse de } \\
\text { I'Arve }\end{array}$ \\
\hline
\end{tabular}

ment affecte les vastes surfaces dénudées en forte pente, assises dans des formations géologiques peu résistantes; la solifluxion, lent fluage vers l'aval des formations schisteuses altérées, imbibées d'eau lors de la fonte des neiges, est également extrêmement active.

2) Les avalanches de fonte canalisées par les axes de drainage ont un double effet : la forte énergie libérée lors de leur écoulement en fait des agents d'érosion particulièrement efficaces; elles sont aussi un agent de transport des débris et jouent le rôle de relai lorsque ces matériaux ont été préparés et déplacés par d'autres processus. En créant des bouchons dans les thalwegs, les culots d'avalanches bloquent les transits de matériaux et accentuent l'instabilité des lits torrentiels en accroissant localement la raideur du profil en long.

3) Les éboulements et chutes de blocs alimentent activement les lits torrentiels. Dans le bassin torrentiel de la Griaz (fig. 1), l'éboulement du "dérochoir des Rognes ", fonctionnel au début du $\mathrm{xx}^{\mathrm{e}}$ siècle, est sans conteste l'exemple le plus spectaculaire de la vallée de l'Arve 
(fig. 2) : les Archives du Service RTM de la Haute-Savoie ont permis d'établir que la fonte d'un petit glacier mort situé en position d'abri libéra d'énormes quantités de débris morainiques. Les chutes estivales incessantes de blocs entre 1888 et 1910 provoquèrent la destruction d'un peuplement de mélèzes; entre 1891 et $1893,40000 \mathrm{~m}^{3}$ de blocs et matériaux de toute granulométrie s'accumulèrent dans le lit du torrent qui s'exhaussa en certains points de 7,40 m (PEIRY, 1988, p. 205-208).

4) Les glissements de terrain sont le processus de recharge sédimentaire le plus actif des torrents de l'Arve. Ils se produisent dans les épaisses formations quaternaires qui nappent les versants, ou dans les schistes et cargneules profondément altérés. Leur activité est sous la dépendance de l'eau, qui modifie les propriétés mécaniques des sols : ainsi, le déclenchement des glissements ou leurs réactivation est souvent liée à des périodes anormalement pluvieuses, ou, si le mouvement est chronique, aux époques de forte imbibation des sols (fonte des neiges).

Le recensement des principaux processus de versant montre que les bassins torrentiels sont des zones de forte production sédimentaire, qui délivrent des débris en abondance et de toute granulométrie. Cependant, à l'échelle d'un bassin torrentiel, elles n'occupent pas forcément de vastes superficies, mais sont le plus souvent très ponctuelles. Les matériaux, stockés temporairement dans les thalwegs, seront repris en charge ulterieurement par les avalanches de fonte ou lors des purges sédimentaires brutales que constituent les transports solides torrentiels.

\subsection{Les transports solides torrentiels}

Les transports solides torrentiels se produisent sous deux formes : le charriage classique, l'eau et les sédiments étant séparés en deux phases distinctes, liquide et solide ; les laves torrentielles, eau et sédiments étant intimement liés en une seule phase visqueuse. Si tous les torrents sont soumis à des phénomènes de charriage, seuls certains d'entre-eux engendrent des laves. Les plus importantes se produisent sous la forme d'une série de pulsations ou "bouffées" séparées par des répits de quelques dizaines de minutes, au cours desquelles les charriages classiques reprennent. La transition d'un état à un autre ne dépend pas uniquement de l'intensité de la crue liquide, mais aussi des matériaux mobilisables: la présence d'argile dans des proportions comprises dans une fourchette relativement étroite est une condition nécessaire (PIERSON, 1980). Qu'ils se produisent par charriage ou sous la forme d'une lave, les transports solides torrentiels sont capables de provoquer le transit de quantités énormes de débris :

1) Les émissaires glaciaires de l'Arve supérieure ont connu des transports solides particulièrement intenses. Ainsi, en juillet 1892, la crue catastrophique consécutive à la rupture d'une poche intra-glaciaire au glacier de TêteRousse, détruisit en partie Saint-Gervais, répandant environ 1000000 de $\mathrm{m}^{3}$ de boues et blocs dans la vallée (Mougin, 1914). En 1938, le torrent chamoniard des Favrands-Tissourds connut une crue si intense qu'un bloc

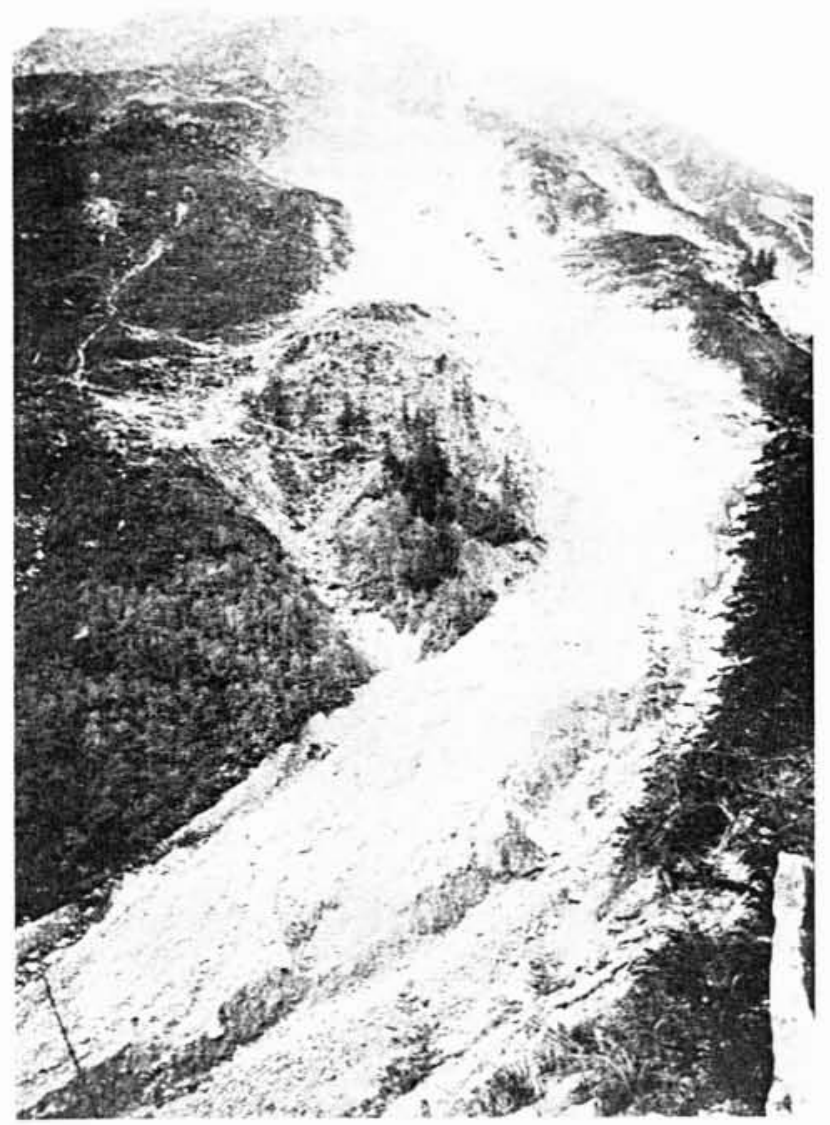

2. Vue du "dérochoir des Rognes " alimentant le torrent de la Griaz, prise en 1895, lorsque l'éboulement était en pleine activité (cliché : collection RTM.74).

cubant plus de $100 \mathrm{~m}^{3}$ parcourut une distance de $350 \mathrm{~m}$ (PeIRY, 1988, p. 212). En dépit de leur intensité, les transports solides des émissaires glaciaires ne semblent se produire que sous la forme de charriages ou écoulements peu visqueux. Ce sont probablement les caractéristiques hydrologiques des cours d'eau glaciaires qui permettent d'expliquer cette particularité: les débits abondants et permanents de la période estivale se chargeant d'exporter en continu les fines contenues dans les dépôts glaciaires, ceux-ci se trouveraient en proportion insuffisante pour que les crues engendrent la formation d'une lave.

2) Les torrents à laves tributaires de l'Arve connaissent eux-aussi des phases de purge sédimentaire. Sur le torrent de la Griaz, la période 1895-1905 fut une décennie de paroxysme torrentiel, des coulées d'une ampleur exceptionnelle se produisant à plusieurs reprises en 1895, 1898 et 1905: Sogreah (1985) évalue à $300000 \mathrm{~m}^{3}$ le volume des matériaux transportés jusqu'à l'Arve pendant cette période; les estimations des Forestiers de l'époque font état d'un chiffre identique, mais pour la seule crue de 1905. Pendant l'été 1964, le torrent d'Armancette, affluent du Bon-Nant connut à deux semaines d'intervalle deux paroxysmes torrentiels qui jetèrent respectivement 60$80000 \mathrm{~m}^{3}$ et $100-120000 \mathrm{~m}^{3}$ de sédiments dans la vallée. 


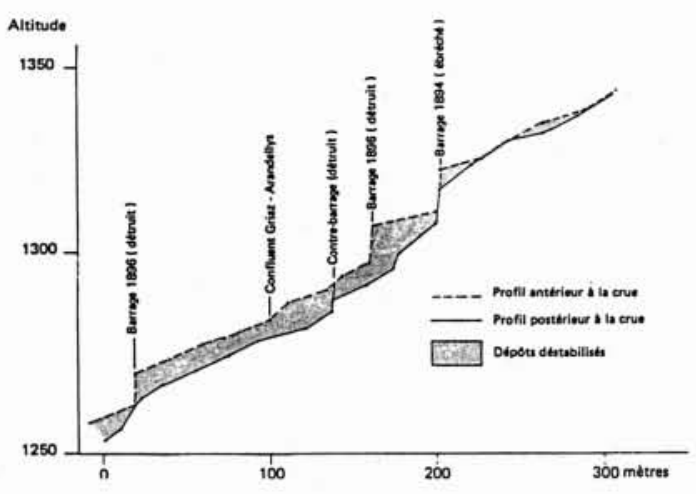

3. Incision du chenal sur le torrent de la Griaz, ravin des Arandellys, après la crue du 20 juillet 1898 ; elle atteignit 10 mètres de profondeur dans plusieurs sections et causa la destruction des barrages (d'après les données du RTM 74).

4. Analyse de la section déstabilisée du Nant-Bourdon : elle met en évidence l'effet de l'incision du chenal sur la stabilité des pentes dominant le lit.

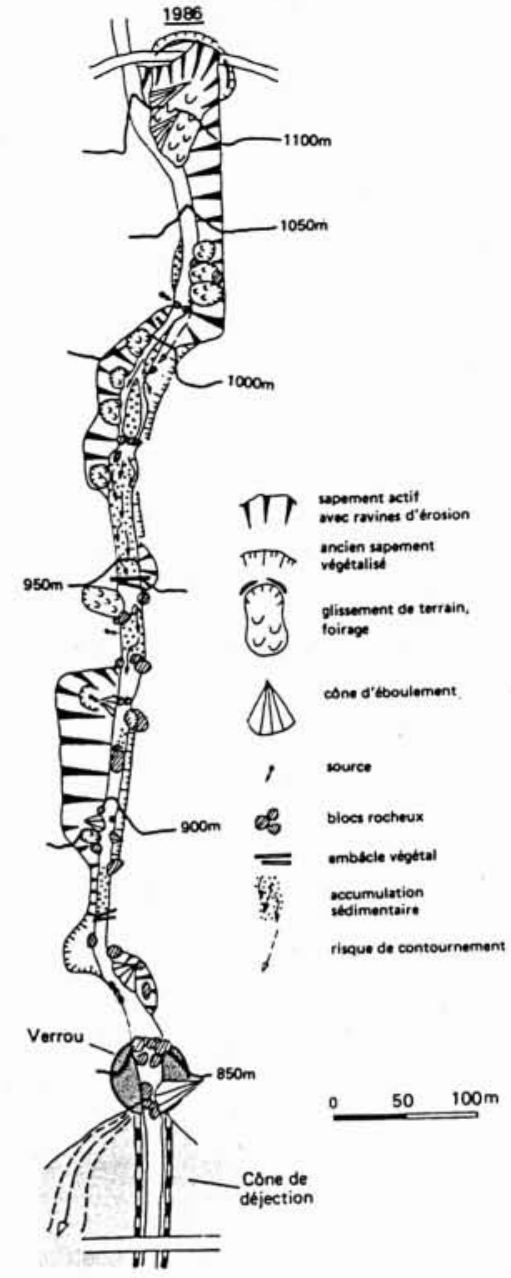

En juillet 1960, une coulée d'ampleur exceptionnelle se produisit sur le torrent de Reninges, l'un des plus actifs de la vallée de l'Arve, obstruant le lit de la rivière et provoquant son débordement sur sa rive gauche. Depuis 1983, le Nant-Bourdon, implanté en bordure du grand glissement de terrain du massif sud de Platé est profondément déséquilibré; ses crues quasi annuelles mobilisent entre $5000 \mathrm{~m}^{3}$ et $10000 \mathrm{~m}^{3}$ et coupent régulièrement le chemin départemental Passy-Servoz. Ces quelques exemples, choisis parmi beaucoup d'autres, illustrent la vigueur du phénomène torrentiel dans la vallée de l'Arve.

Les épisodes de purge sédimentaire ont une incidence considérable sur la géomorphologie des lits torrentiels :

1) A la faveur de substitutions de charge lors d'élargissements du lit ou de diminutions locales de la pente, en arrière d'embâcles organiques, les transports solides torrentiels abandonnent une partie de leur charge. Ces accumulations sédimentaires prennent la forme de levées latérales ou d'accumulations de chenal. La totalité des sédiments peut même parfois être stoppée, lors d'une larve avortée; il s'agit là de dépôts temporaires, souvent très instables, qui alimenteront des transports solides ultérieurs. Les cônes de déjection, zone de brutale décroissance de la pente constituent la principale aire de stockage, souvent définitive des matériaux détritiques torrentiels; l'exhaussement du chenal favorise les débordements et l'épandage des coulées sur le cône.
2) Les phénomènes d'incision se produisent dans toutes les sections du torrent, après destruction du pavage stabilisant le profil en long : en août 1895 , sur le torrent de la Griaz, des abaissements du lit supérieurs à 9 mètres furent observés (fig. 3). Ils favorisèrent la mobilisation d'énormes quantités de débris jusqu'alors stockés dans le chenal. L'incision touche également les cônes de déjection, les laves les plus énergiques provoquant un auto-curage du lit: les crues affouillantes de juillet et août 1895, sur le torrent de la Griaz, recalibrèrent le chenal sur une longueur du cône d'environ $2 \mathrm{~km}$, remettant en mouvement environ $200000 \mathrm{~m}^{3}$ de matériaux. Ils obstruèrent le lit de l'Arve, créant une petite retenue temporaire, qui subsista plusieurs semaines. Cet exemple illustre le rôle trop souvent négligé du cône de déjection, dans l'alimentation des transports solides torrentiels.

3) L'incision du chenal est accompagnée d'une intense attaque des berges qui déstabilise les versants qui dominent le lit : ainsi, sur la section déstabilisée du Nant-Bourdon, l'attaque de la base des glissements surplombant le thalweg entretient leur déséquilibre, par suppression de la butée de pied: les sapements de berge provoquent également le foirage des versants, par dépassement de la limite d'équilibre des terres (fig. 4). Dans une certaine mesure, l'instabilité des lits torrentiels conditionne donc l'évolution des versants et contrôle leur production sédimentaire. 


\subsection{Les facteurs du déclenchement des transports solides torrentiels}

Le déclenchement des transports solides torrentiels a pour double origine :

1) l'ampleur de la crue liquide qui dépend des conditions hydro-météorologiques sévissant dans les bassins versants ;

2) la disponibilité en matériel susceptible d'être prise en charge, qui dépend de l'intensité de la fourniture sédimentaire par les processus de versant.

CAINE (1980) a mis en évidence l'existence d'un seuil intensité de la précipitation/durée, contrôlant la production des transports solides torrentiels. Lors des orages estivaux, la rapide concentration des écoulements sur les terrains dénudés des bassins de réception favorise des crues liquides brutales, fortement énergiques. Sur les émissaires glaciaires, soumis en plus à des vidanges de poches intraglaciaires ou de lacs supra-glaciaires, les crues liquides prennent la forme de débâcles qui exercent un effet de chasse sur les sédiments. Cependant, deux observations permettent d'affirmer qu'une forte crue liquide n'est pas une condition suffisante pour que se déclenchent d'intenses transports solides: on note d'importantes variations du comportement de torrents voisins, où les conditions environnementales paraissent identiques et où sévissent les mêmes conditions météorologiques; la fréquence des purges sédimentaires d'un bassin torrentiel donné est susceptible de varier très rapidement dans le temps, sans pour autant que le facteur météorologique change.

La synthèse des données recueillies sur les torrents de l'Arve montre que le degré de stabilité des bassins torrentiels est étroitement lié aux fluctuations du bilan sédimentaire dans les thalwegs. Lorsqu'il se produit un excès d'accumulation sédimentaire dans toute ou partie du lit d'un torrent, sa susceptibilité à produire des transports solides majeurs pour des précipitations d'intensité donnée est plus forte que sur un torrent où l'accumulation est moindre. Ainsi, il apparaît que l'intensité de la fourniture sédimentaire aux thalwegs conditionne la fréquence et l'ampleur des transports solides, donc l'activité torrentielle: sur le torrent de la Griaz pendant la décennie 1895-1905, la succession d'énormes laves eut pour origine les apports de matériaux surabondants délivrés par le «dérochoir des Rognes ». Ils accrurent l'instabilité du système torrentiel qui se manifesta par une augmentation de la fréquence et de l'intensité des purges sédimentaires, sans pour autant que les précipitations soient systématiquement exceptionnelles. Dans les cas extrêmes, certaines bouffées de lave peuvent même se produire plusieurs heures après l'arrêt des précipitations : ce fut le cas, lors de la crue exceptionnelle de la Ravoire des Ares en 1981 (L. de CRÉCY, communication personnelle), ce qui indique un phénomène d'autoentretien des transports solides par renouvellement continuel de la charge et le maintien du système en état de déséquilibre permanent.

L'analyse de la dynamique des sédiments dans les torrents de l'Arve et la mise en évidence de leur influence sur l'activité des transports solides ont permis d'établir une classification fondée sur le type et l'intensité de la fourniture sédimentaire alimentant les thalwegs:

1) sur la plupart des torrents, le remblaiement des chenaux est relativement lent. C'est l'occurrence de précipitations exceptionnelles qui est le principal facteur contrôlant la production des transports solides torrentiels. Elles provoquent des ruptures temporaires de l'équilibre du système torrentiel, suivies de répits de longue durée;

2) sur d'autres torrents, le remblaiement des thalwegs est très rapide et fonction de processus de versants indépendants de l'évolution du lit torrentiel. Les transports solides de grande ampleur sont fréquents, sans forcément être liés à des précipitations exceptionnelles. Dans ce cas, c'est la dynamique de la fourniture sédimentaire aux thalwegs qui exerce un contrôle prépondérant sur la production des transports solides. Ce fut le cas entre 1895 et 1905 , sur le torrent de la Griaz, lors de sa période de crise morphodynamique afin d'évacuer la charge surabondante fournie par le «dérochoir des Rognes ". Avec l'arrêt des chutes de blocs, la fréquence des transports solides majeurs a diminué, ce qui indique le retour rapide vers un état plus stable ;

3) sur une troisième catégorie de torrents, le remblaiement des thalwegs est également très rapide, mais est entretenu par des processus de versant dont l'activité dépend de l'évolution du chenal (incision du lit, sapements de berge...). La déstabilisation des versants dominant le thalweg accélère la recharge sédimentaire et favorise l'auto-entretien de l'instabilité torrentielle. Sur ce type de torrent, le retour à la stabilité est extrêmement difficile, en dehors de toute intervention anthropique visant à maîtriser son activité.

\section{L'impact des interventions de l'homme sur la dyna- mique torrentielle}

L'homme intervient sur les torrents dans le but de contrôler la dynamique des flux hydriques et sédimentaires. L'aménagement des bassins versants a été développé dans la montagne, mais également sur les cônes de déjection.

\subsection{Les travaux de correction torrentielle dans la montagne}

Les interventions de l'homme se produisent à la fois sur les pentes des bassins de réception et dans le chenal d'écoulement :

1) sur les versants, les travaux visent à ralentir la fourniture sédimentaire aux thalwegs et à diminuer la vitesse de concentration des eaux lors des épisodes d'intenses précipitations. Le reboisement a été l'une des actions clé engagée sur les torrents de l'Arve par le service RTM de la Haute-Savoie. Les conditions écologiques difficiles des zones d'altitude, l'intensité des contraintes géomorphologiques ont rendu ces interventions aléatoires: sur les 


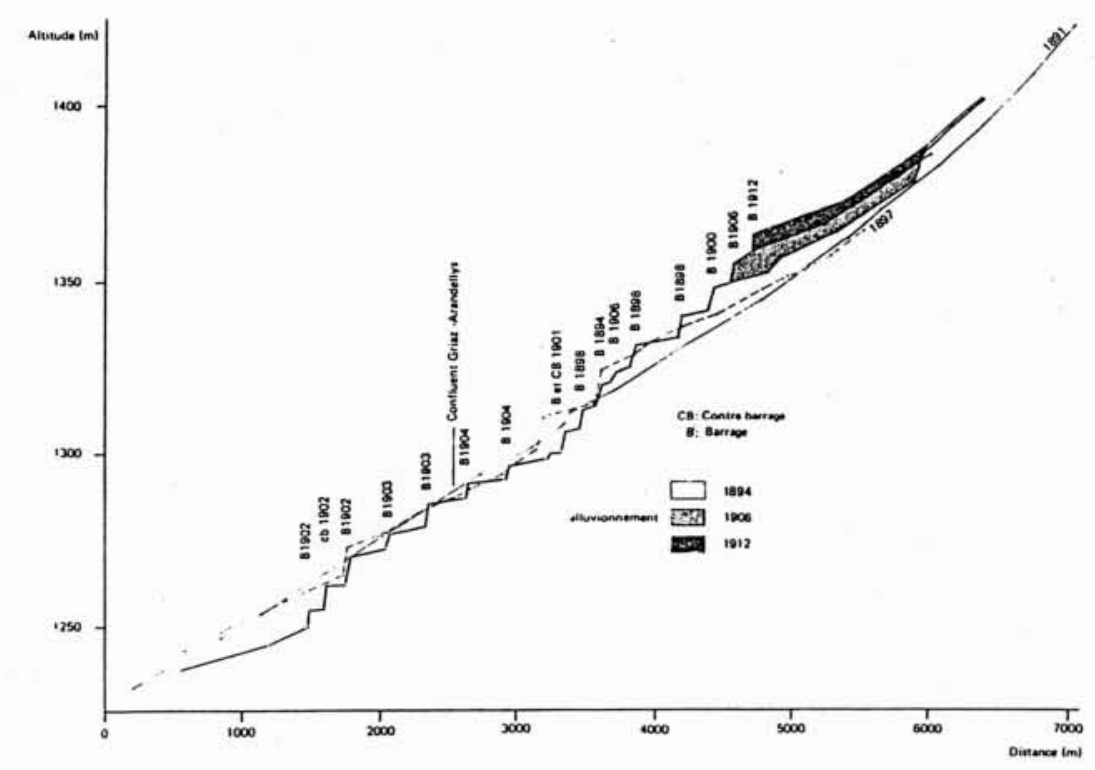

5. Accumulation sédimentaire après la construction des barrages dans la section aménagée du torrent de la Griaz: en 1973, le volume des dépôts était d'environ $100000 \mathrm{~m}^{3}$; actuellement, plusieurs barrages sont enfouis sous les sédiments (d'après les données du RTM 74).

versants stabilisés, les plantations se sont développées avec succès et limitent les érosions superficielles ; sur les versants les plus pentus, soumis à d'intenses ravinements et balayés par les avalanches, les plantations n'ont pu se développer malgré les efforts mis en œuvre par les forestiers. Ce constat montre que des contraintes géomorphologiques réduites étaient le préalable à toute installation de la végétation, le reboisement consistant alors simplement à accélérer le processus naturel de revégétalisation ;

2) dans le chenal d'écoulement, les interventions des Forestiers ont essentiellement consisté à édifier des barrages de correction (fig. 5). Ils avaient pour objectifs : de stabiliser les pieds des glissements dominants le thalweg; de fixer le profil en long, afin d'empêcher les affouillements ; de retenir les matériaux détritiques dans la montagne par l'édification d'aires de stockage ; de dissiper l'énergie des crues en augmentant artificiellement les pertes de charge. Les barrages ont souvent été partiellement, voire complètement détruits lors de violentes crues affouillantes. Leur rupture est d'autant plus redoutable qu'ils stockent un volume considérable de matériaux (fig. 5) : en 1973, sur le torrent de la Griaz, l'accumulation sédimentaire dans la zone des barrages était estimée à $100000 \mathrm{~m}^{3}$. Cette masse de débris constitue un danger potentiel pour la vallée et rend impératif le maintien des ouvrages en état. Leur efficacité est souvent amoindrie par leur enfouissement sous les dépôts; en outre, cet engravement accroît les risques de contournement des barrages et de déstabilisation de l'ensemble du système de correction. Des curages du chenal sont alors nécessaires dans les secteurs accessibles sans trop de difficultés.

\subsection{L'aménagement des cônes de déjection}

Plusieurs types d'aménagements ont été développés sur les cônes de déjection :

1) les endiguements constituent le moyen traditionnel de protection contre les crues torrentielles. Ils existent au moins depuis le XVII ${ }^{e}$ siècle dans la vallée de l'Arve et ont longtemps opposé une résistance dérisoire face à la puissance des écoulements de lave ;

2) plus récemment, des travaux de canalisation du chenal ont été entrepris sur plusieurs torrents de la vallée. Ils empêchent les débordements lors du passage des bouffées de lave et facilitent le transit des sédiments jusque dans le lit de l'Arve ;

3) en 1968, un bassin d'accumulation a été construit sur le torrent de Reninges, immédiatement en amont de la confluence avec l'Arve. D'une capacité de $25000 \mathrm{~m}^{3}$, il a pour fonction le blocage des laves, afin d'empêcher l'obstruction du lit de l'Arve. Il a été curé à onze reprises depuis sa construction, ce qui a permis l'évacuation de plus de $35000 \mathrm{~m}^{3}$ de matériaux.

Peu efficaces avant 1950, en raison du manque de moyens techniques, les curages du chenal ont été largement développés sur les cônes de déjection, depuis cette date. Dans un premier temps, ils ont été effectués après des crues fortement chargées, afin d'assurer le déblaiement du chenal et d'éviter des débordements ultérieurs.

Avec la pénurie de matériaux alluvionnaires consécutive à l'interdiction d'extraire dans le lit de l'Arve, les curages sont devenus une véritable industrie : 
1) sur le torrent de la Creusaz, émissaire du glacier des Bossons, $60000 \mathrm{~m}^{3}$ ont été extraits en 1982-83, puis à nouveau $20000 \mathrm{~m}^{3}$ en 1985 . Le chenal, jusqu'alors suspendu au-dessus du cône, s'est profondement enfoncé et tout risque de débordement est actuellement écarté ;

2) sur le torrent de la Griaz, les curages ont permis l'extraction de plus de $100000 \mathrm{~m}^{3}$ de dépôts depuis 1981 et l'incision du chenal s'est développée bien au-delà des zones de prélèvement, par érosion régressive.

L'impact des curages sur la dynamique de la recharge sédimentaire de l'Arve est difficile à cerner: dans un premier temps, les ajustements du profil en long accroissent les transports solides; à plus long terme, l'évolution des transports solides dépendra de la poursuite ou non des extractions. Cependant, comme on l'a montré, il apparaît que le curage des cônes de déjection, en maintenant un chenal parfaitement calibré, suppriment une importante source de débris alimentant les transports solides les plus énergiques.

\section{Conclusion}

L'analyse du comportement des sédiments sur huit bassins torrentiels a permis d'appréhender la dynamique des flux de matériaux. En particulier, elle a mis en évidence le rôle fondamental des accumulations sédimentaires se produisant dans les thalwegs sur le déclenchement des transports solides: de multiples processus de versant assurent la préparation des matériaux détritiques et se relaient pour les acheminer jusqu'aux lits torrentiels, où ils sont temporairement stockés; ces accumulations diminuent la stabilité du système et accroissent les risques de purge sédimentaire de grande ampleur. L'intensité de l'activité torrentielle résulte d'un équilibre complexe entre les apports détritiques et les flux d'eau :

1) si la fourniture sédimentaire alimentant le chenal est surabondante, les purges sédimentaires se succéderont à un rythme rapide indiquant la profonde instabilité du système. Dans ce cas, la fourniture sédimentaire est le facteur principal de l'activité torrentielle, tout flux d'eau suffisamment énergique permettant le déclenchement des transports solides ;

2) en l'absence de forts apports détritiques, les purges sédimentaires sont liées aux flux d'eau d'ampleur exceptionnelle. C'est alors l'occurrence des conditions hydrométéorologiques favorables, par définition rares, qui sera le principal facteur du déclenchement des transports solides.

Actuellement, la plupart des torrents de l'Arve ont une activité modérée et les transports solides majeurs sont liés à l'occurrence des précipitations exceptionnelles.

La réussite de la politique d'aménagement des bassins torrenticls entreprise depuis la fin du $\mathrm{XIX}^{\mathrm{C}}$ siècle est sans aucun doute l'une des causes de la baisse d'activité observée depuis un siècle:
1) les interventions anthropiques dans la montagne ont favorisé le blocage de la charge et empêchent les transits de matériaux. Ce déficit d'apport concerne avant tout les sédiments les plus grossiers, qui ne peuvent être repris en charge par les épisodes hydro-météorologiques courants ;

2) la protection des cônes de déjection, et en particulier les curages ont fortement artificialisé la réinjection sédimentaire dans l'Arve. Ici encore, il est très probable que les sédiments grossiers sont les principaux touchés par l'aménagement des cônes.

En définitive, il apparait que les torrents de l'Arve constituent des systèmes tout aussi perturbés que l'Arve ellemême. Les interventions de l'homme sur les torrents ont fortement modifie la dynamique des sédiments assurant la recharge de l'axe de drainage de la vallée.

Remerciements : Cet article est tiré d'une étude réalisée à la demande du Service régional pour l'aménagement des eaux, Rhône-Alpes. Nous tenons à remercier M. J.P. Merle, chef du SRAE et son adjointe Mme Th. SANCHIS qui ont été les initiateurs. Nos remerciements vont aussi a M. P. Cogoluenhes, Chef du Service de restauration de terrains en montagne de la Haute-Savoie, qui nous a accueilli dans ses locaux et ouvert sans réserve ses archives.

\section{Bibliographie}

Blanchet, C., M. Brissaut (1968), Evolution du lit du Drac dans la traversée de Grenoble. $10^{\circ}$ Journées de l'hydraulique, Paris, 3-5 juillet 1968, SHF, t. 2, question 5, rapport $8,7 \mathrm{p}$.

Blanic, M., G. Verdet (1975), Quelques travaux de correction sur le cours de l'Isère. La Houille Blanche, 2-3, p. 191-198.

CAINE, N. (1980), The rainfall intensity-duration control of shallow landslides and debris-flows. Geografiska Annaler, 62-A, p. 23-27.

Mougin, P. (1914), Les torrents de Savoie. Imp. Générale, Grenoble, $1251 \mathrm{p}$.

PEIRY, J.-L. (1987), Channel degradation in the middle Arve river (France). Regulated rivers, 1-2, p. 183-188.

PEIRY, J.-L. (1988), Approche géographique de la dynamique spatiotemporelle des sédiments d'un cours d'eau intra-montagnard: lexemple de la plaine alluviale de l'Arve (Haute-Savoie). Th. Univ. Lyon 3, $378 \mathrm{p}$.

Pierson. T. C. (1980), Erosion and deposition by debris flows at Mont. Thomas, New-Zealand. Earth. Surf. Processes and Landforms. 5, p. 227-247.

SOGREAH, (1985), Etude hydraulique du franchissement de la Griaz par la voie express le Fayet-les-Houches. Rapport d'ingénieur non publié, $\mathrm{n}^{\circ} 46.0209,60 \mathrm{p}$. + plans.

Tricart, J. (1960), Mécanismes normaux et phénomènes catastrophiques dans l'évolution des versants du bassin du Guil (Hautes-Alpes, France). Zeitschrift für Geomorph., 5/4, p. 277 301. 